\title{
MODERN COMPLEX METHODS OF REAGENT-FREE PROCESSING OF LIQUIDS FOR SUBSEQUENT NON-CONTACT PARALLEL MONITORING IN THE PROCESS OF AERIAL PHOTOGRAPHY OR USING UNMANNED AERIAL VEHICLES
}

Yevhen Zharkov

Master in Computer Science. Open International University of Human Development 'Ukraine'. Ukraine. Kyiv.

The use and creation of a complex system of contactless impedance-resonance monitoring of the state of liquids can be considered an integrative demonstration of the perfect final result in accordance with the criteria of the Theory of Inventive Problem Solving.

Only integrative way, since the technology is an integrated and highly efficient compilation of a whole range of system technologies, including nanotechnological solutions in several frontier technological fields. 


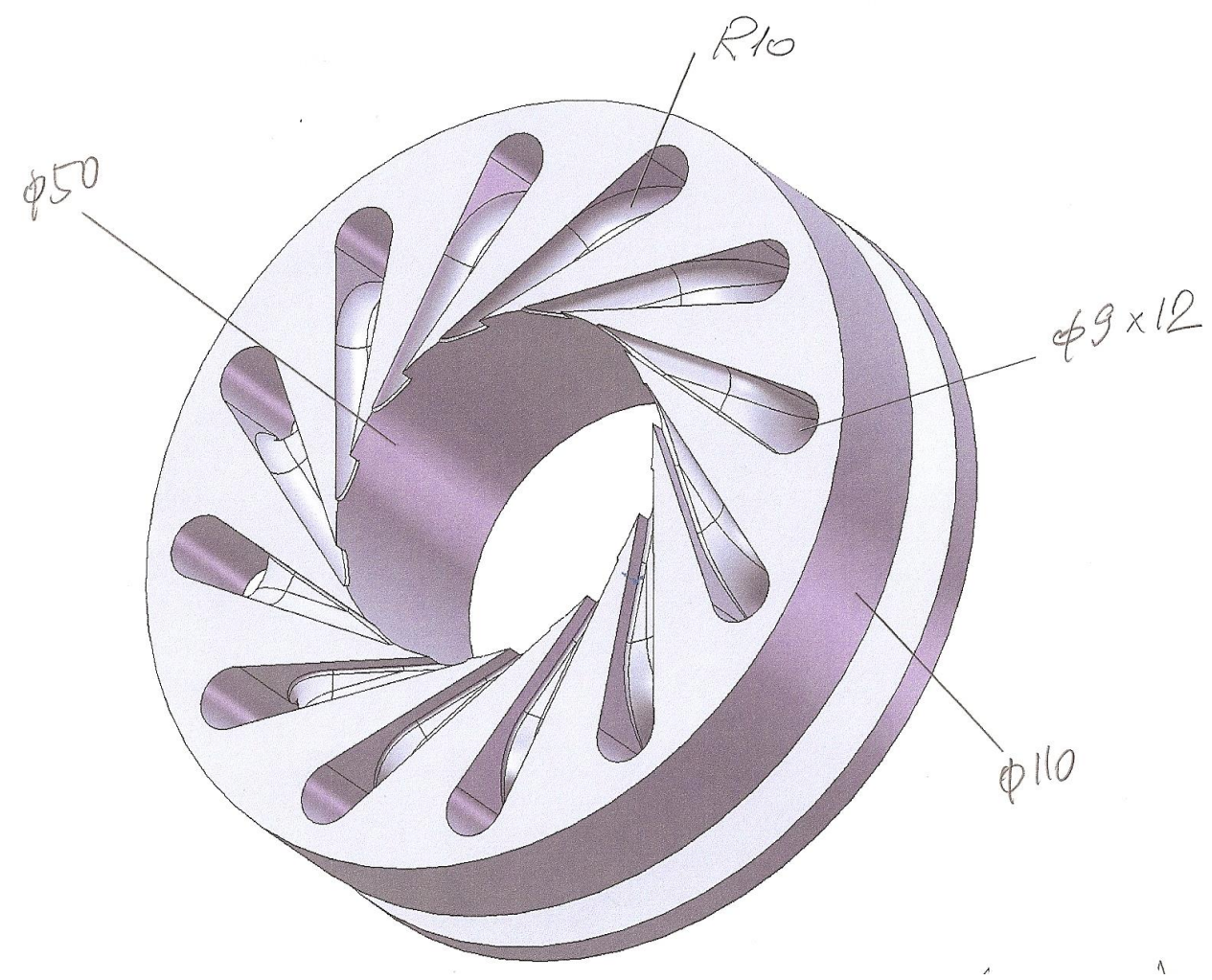

Figure 1. One of the basic elements for the formation of a system for the treatment of liquids and solutions is a vortex generator

The quality of technological solutions and liquids, as a subject of targeted innovation, has acquired particular importance in our time because of many deep-seated global processes, including global warming and the use of biochemical additives to basic technological solutions, and the use of genetically modified materials and compounds other risk factors, such as a steady increase in the use of chemical fertilizers.

As specialists, technologists and environmentalists emphasize, in order to systematically control the quality level of any technological solutions, emulsions and blends, especially products of composite or multicomponent processes, traditional control methods and especially non-contact monitoring are no longer enough. 
To control the quality of liquids of such a level that meets the dictates of time, a system of sensors operating on the principles of magnetic resonance was invented.

It makes sense to dwell on the essence of this method:

The method involves the creation of an alternating electro-magnetic field in the space in which the sample under study is located. This field is a mediator or connecting element between the resonant circuit and the sample under test.

On the one hand, the resonant circuit is an emitter of this field, on the other hand, it is an acceptor (sensitive element) of the changes in the electromagnetic field that the sample under test makes.

Even in the absence of the test sample, the alternating electromagnetic field generated by the sensor solenoid is the aggregation of two electromagnetic fields that vary in anti-phase with each other. One field is generated by a change in the magnetic induction of the solenoid of the controlling element of the system and has a vortex electric field (Maxwell-Faraday equation).

Another is caused by a change in the electric field created by the potential difference between the outermost coils of the specified solenoid (if the sample is placed inside the solenoid) or the potential difference between the coil closest to the surface of the measured element and the measured element itself (if the element is opposite the solenoid end) and has the effect of a vortex magnetic field (Ampère's circuital law with Maxwell's correction).

Under the influence of the specified external AC magnetic field in the sample under test, depending on its nature, electric phenomena such as linear and eddy conduction currents, linear and eddy bias currents (caused by dielectric polarization), as well as linear and eddy ion currents (ordered movement of ions). 
In accordance with the principle of superposing fields, these electrical phenomena introduce distortions into the indicated external alternating electromagnetic field. These distortions are perceived by the solenoid of the controlling element - the resonant sensor.

The resonant circuit of the specified control element, which includes this solenoid, under the influence of these distortions, changes its behavior in the same way as if such additional elements were added to it: such as a capacitor, inductance, and a resistor.

The combination of these specified additional capacitive, inductive and active resistances is an additional impedance introduced into the system by the test sample, this additional impedance measures the non-contact control element the resonant sensor.

Changes in the parameters of the resonant circuit are reflected in changes in its amplitude-frequency characteristic, with this change, the resonant frequency and amplitude of the specified resonant circuit, which is part of the controlling element of the system. Investigating the magnitude and nature of these changes, one can judge the impedance of the sample under study.

A device for magnetic resonance quality control of technological solutions, including water or aqueous solutions, is a section of the pipeline on which an circular sensor is installed on the outer surface of the pipe, connected to a power source and a control and amplification and identification system.

The system includes an optional transmitting device, for transmitting an amplified and identified signal to the aerial camera operator's console or to an unmanned aircraft processor or even to a mobile phone.

The system is tuned to a reference sample of water or any other liquid, taking into account all local conditions. 
The system sensor records any change in the state of water or any other liquid, the signal is identified and transmitted to the aerial camera operator's console, computer monitor, or mobile phone.

The frequency of control tests may vary with settings ranging from one test at 0.1 second to one test at 1 minute.

Among the main parameters of the sensor for the resonant control of the quality of water or other types of process liquids mentioned above, accuracy and sensitivity should be referred. The major parameter is sensitivity, it is an ability to distinguish, fix and identify the difference equivalent to 0.000001 grams per liter.

To perform similar control operations based on existing technology, it requires specially trained qualified staff, the cost of working hours of which is more than $\$ 35$.

Staff can not perform more than one test per day and during the period between tests the state of water or liquid material is not monitored, which leads to emergency situations.

The time from the moment of detection of the problem to the elimination is increased due to the fact that there is no possibility of permanent control over the quality of water; the transfer of information is not operational, which also increases the time to resolve emergencies. 
The proposed method of complex, extremely remote control of the parameters of the state of a material liquid object can serve as an equivalent of the ideal end result and includes:

- the formation of a three-dimensional spatial system in which the controlling element covers the controlled element;

- the alignment of the centers of symmetry of the cross-sections of the controlling and controlled elements of the specified three-dimensional spatial system;

- stabilization of a uniform gap between the outer surface of the test element and the controlling element of the specified three-dimensional spatial system;

- the formation around and inside the volume occupied by the controlling element of an energetically saturated space in the form of a variable electromagnetic field with a controlled and regulated intensity;

- the impact of the specified alternating electromagnetic field on the controlled element and the induction in it of linear and eddy conduction currents, linear and eddy bias currents and linear and eddy ion currents;

- identification and comparative analysis of distortions arising in the specified alternating electromagnetic field and their perception of the controlling element by the resonant circuit as additional elements, - capacitor, inductance and resistor

- fixing the levels of arising distortions and changes in the parameters of the specified resonant circuit in part of its amplitude-frequency characteristic in the form of a change in the resonant frequency and amplitude and an estimate on their basis of the impedance of the controlled element determining the state parameters of the material object; 
In addition, the proposed method for remote monitoring of state parameters of a liquid material object, mainly in the form of a liquid or a mixture of liquids or a mixture of liquids and gases or in the form of aerosols, includes:

- the formation of a three-dimensional spatial system in which the controlling element encompasses a controlled element placed in the shell that defines and supports the geometric shape of the controlled element;

- the alignment of the centers of symmetry of the cross-sections of the controlling and controlled elements of the specified three-dimensional spatial system;

- stabilization of a uniform gap between the outer surface, placed in the specified shell, the controlled element and the inner surface of the controlling element of the specified three-dimensional spatial system;

- the formation around and inside the volume occupied by the controlling element of an energetically saturated space in the form of an alternating electromagnetic field with controlled and adjustable characteristics;

- excitation and induction in the volume, placed in the specified shell, of a controlled element connected with the resonant circuit of the controlling element by means of an alternating electromagnetic field — linear and eddy conduction currents, linear and eddy bias currents and linear and eddy ion currents;

- identification and comparative analysis of the distortions arising in the specified alternating electromagnetic field and ensuring that the controlling element perceives the resonant circuit as additional elements - the capacitor, inductance, and resistor; 
- fixing the levels of arising distortions and changes in the parameters of the specified resonant circuit in part of its amplitude-frequency characteristic in the form of a change in the resonant frequency and amplitude and an estimate on their basis of the impedance of the controlled element determining the state parameters of the material object;

- consistent, equal to the number of composite components in a material object placed in the specified shell, changing the values of the characteristics of the energetically saturated space in the form of a variable electromagnetic field formed around and inside the volume occupied by the controlling element;

- identification and comparative analysis of the distortions arising in the specified alternating electromagnetic field and ensuring that the controlling element perceives the resonant circuit as additional elements - the capacitor, inductance, and resistor;

- sequential fixation of arising distortion levels and changes in the parameters of the specified resonant circuit in terms of its amplitude-frequency characteristic in the form of a change in the resonant frequency and amplitude and an estimate on their basis of the impedance of the monitored element determining the state parameters of the composite components of the monitored material object

The energetically saturated space in the proposed technical solution is an alternating electromagnetic field, which is characterized by frequency and intensity.

Control and adjustment of variable electromagnetic field parameters allows to provide the necessary accuracy and flexibility of measurement and control material objects parameters in their various variations;

Since proposed method has no difference in the perception of solid or soft materials, liquid or solid materials, a mixture of liquid and gaseous materials, it 
is enough to bind and adjust the method to different conditions to minimize technological factors.

If we look at the problem of controlling liquid materials and products and technological solutions, mixtures and emulsions, from a practical point of view, to apply the latest methods of remote monitoring or quality control without contact, we can assume that control accuracy and sensitivity of sensors entirely depends on the number of components in the mixture liquids.

Regarding this information the transition of all industries, even partially, to systemic multi-component process boxing or packaging, will minimize the number of simultaneously monitored or controlled components, which will significantly improve the accuracy and quality of control and open the way for innovative measurement technology without direct contact with measured liquid from significant distances, as in case with aerial photography or equivalent unmanned methods of real-time monitoring.

It is important to mark some features of the application of these processing methods for various situations and technological transitions:

1. Liquids processing and solutions containing organic materials in combination with heavy metals

Combined treatment is used to separate a liquid into fractions with a high concentration of organic substances and a liquid with heavy metal ions, where formed foam characteristics and parameters changes during compressed air pressure changes.

A liquid with a high concentration of organic substances is separated from the foam and after removing the foam, a liquid containing predominantly heavy metal ions remains. 


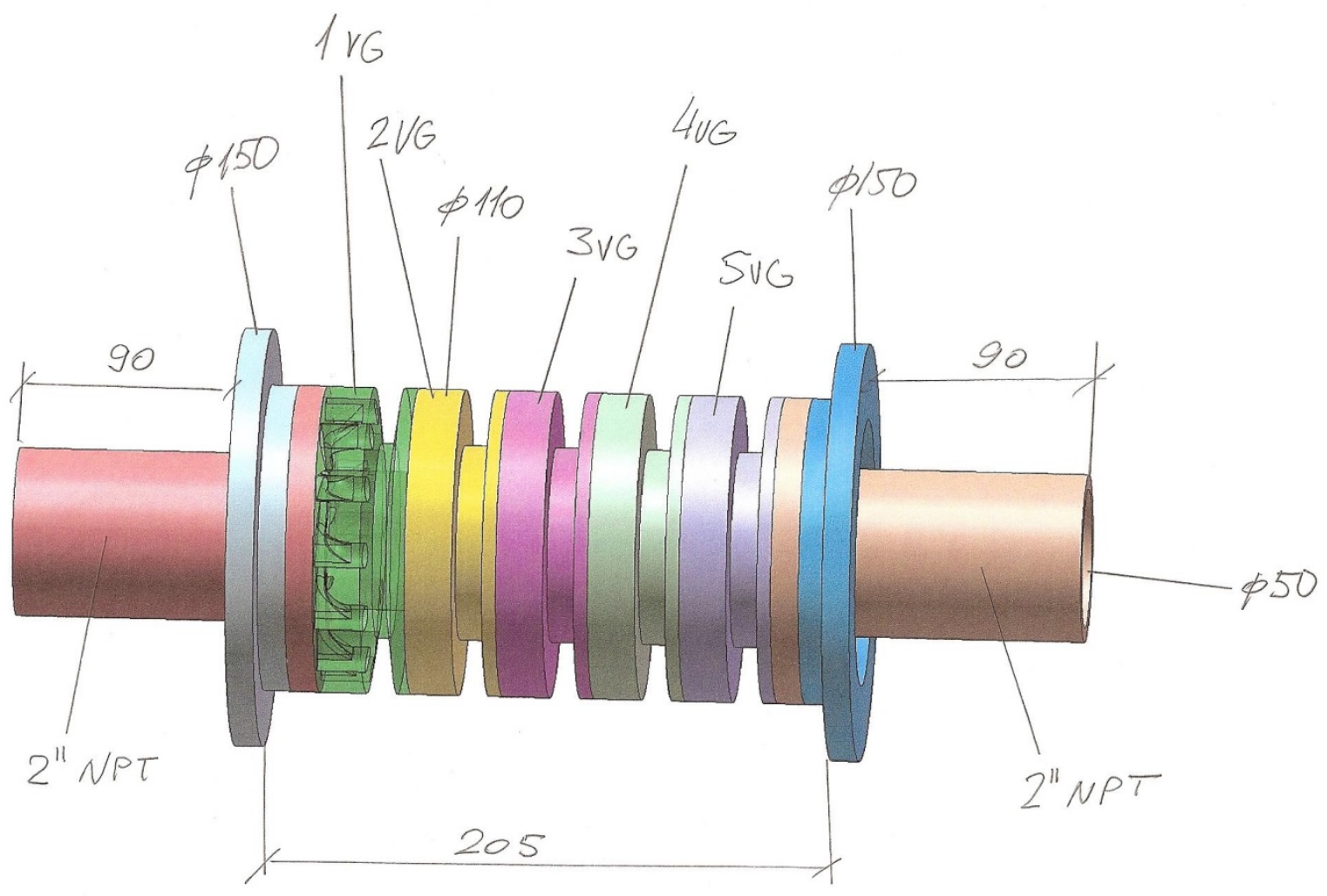

Figure 2. System for on-line formation of a vortex tube in a pipeline, consisting of 5 consecutive vortex generators

2. Liquids processing with an acidic background containing heavy metals in combination with organic acids

Usually, such liquids contain complex contaminants (TSS, TDS, BOD, COD) and to extract heavy metals, it is necessary to separate the fractions of contaminants first.

Combined treatment is used to separate a liquid into fractions with a high concentration of organic substances and into a liquid with heavy metal ions, in where characteristics and parameters of the formed foam change when the pressure of compressed air changes. 
A liquid with a high concentration of organic substances is separated from the foam and after removing the foam, a liquid containing predominantly heavy metal ions remains.

In addition, active homogeneous treatment with air leads to the primary formation of oxides, which simplifies the process of further processing of water or an aqueous solution.

3. Treatment of liquids with an alkaline background containing heavy metals in combination with heavy metal salts

As a rule, such liquids contain complex contaminants (TSS, TDS, BOD, COD) and to extract heavy metals, it is necessary to separate the fractions of contaminants first.

Combined treatment is used to separate a liquid into fractions with a high concentration of organic substances and into a liquid with heavy metal ions, where characteristics and parameters of the formed foam change when the pressure of compressed air changes.

A liquid with a high concentration of organic substances is separated from the foam, and after removing the foam, a liquid containing predominantly heavy metal ions remains.

4. The formation of eddy cutters for cleaning surfaces

The formation of a vortex tube allows you to create active cavitation on the surface being cleaned and washed, which increases the cleaning and washing efficiency by $3-5$ times and reduces the water consumption required for these technological operations by $1.8-2.5$ times. 


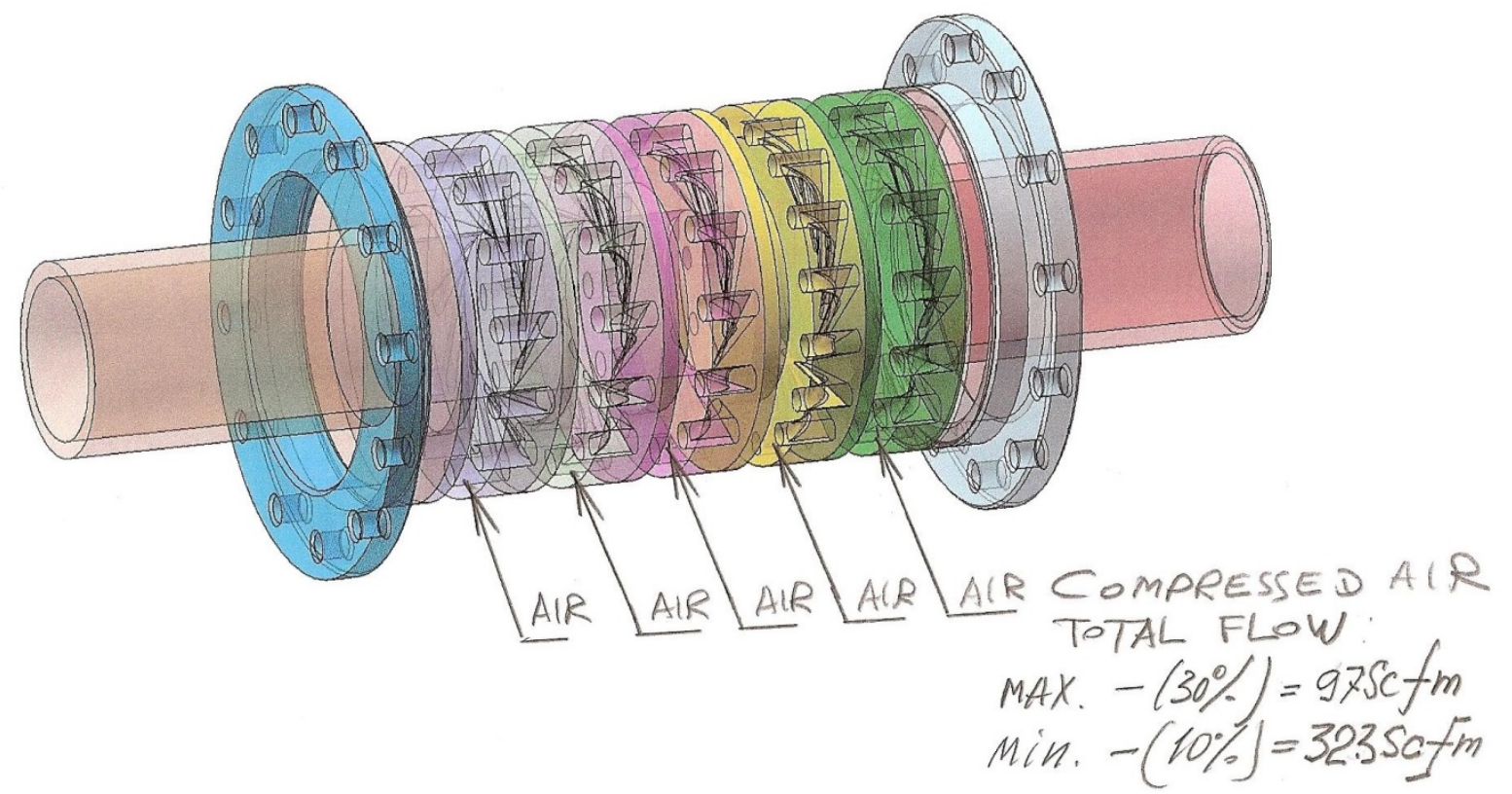

Figure 3. System connection diagram for forming conditions in a pipe for creating a vortex flow - a vortex pipe

5. Pools for washing large parts

In this case, devices for foam formation (foam generators) are used to create a uniform bubbling mode with minimum gas bubbles and raising the level of liquid in the pool, which occurs during the operation of foam generators, which makes it possible to reduce by $25 \%$ to $35 \%$ the amount of contaminated water

6. Saturation of liquids with gas

The use of foam generators or their structural equivalents allows to increase the efficiency and reduce the cost of saturation processes of various liquids with different gases.

At the same time, the technology of foam generators makes it possible to have complete control in real time of the geometry of compressed gas bubbles during the saturation process. 
7. Cold rolling in metallurgical engineering

Water or an aqueous solution can be modified by introducing a stream of bubbles of compressed gas (for example nitrogen), with a size of no more than 25-50 microns.

When gas is introduced into a liquid, it lowers its temperature and then when it comes into contact with the cooled surface and when the shells of air bubbles break, gas expansion and more substantial cooling occur.

This allows you to reduce energy costs for cooling in 2 - 3 times and significantly increase the efficiency of the cooling process.

The cooling process can be carried out at the same cost of energy in parallel with the process of washing or cleaning the cooled surfaces.

8. Classic flotation technology

Devices for the foam formation (foam generators) can be effectively used to generate uniform in size air bubbles.

At the same time, the adjustment of technological modes is considerably simplified, since it is carried out only by adjusting the parameters of compressed air.

This significantly reduces energy costs and improves the quality of cleaning.

9. Combination flotation technologies with dynamic foaming

Foam generators can be used to create a combined flotation process, where at the first stage, foam is formed with a high air pressure (6-8 bar), and after separation, at low pressure ( $0.5-1.5$ bar), treatment is carried out by forming a uniform flow of air bubbles

10. Forced liquid and contaminants separation technologies when small concentrations of detergents are introduced into the cleaned fluid 
In many cases, the fluid to be cleaned does not contain foaming substances. In this case, it is advisable to introduce a small amount of detergent into the liquid (no more than 25 milligrams per liter of the liquid being cleaned).

The active foam, which is formed during the operation of the foam generator, makes it possible to significantly reduce the concentration of contaminants before switching the foam generator to the production of air bubbles and, accordingly, switching to the classical flotation mode.

\section{Technologies of dynamic mixing of various liquids}

When a liquid is supplied to the foam generator, instead of air, and a stream coming out of the foam generator into the volume of another liquid is introduced, an effective uniform dynamic mixing process takes place, which is 5 to 7 times more effective than the mechanical mixing process.

12. Water saving technologies for technological baths

Foam generators use in various technological baths, the effect of raising the level of water or any other liquid in the bath, allows you to lower the level of water or other technological solution in the bath by $25-30 \%$.

This saves technological solutions in $10-15 \%$, with a small consumption of energy for the preparation of compressed air.

\section{Fine mist formation technologies}

Fog appears above the surface of the vortex tube formed during the operation of the foam generator, consisting of air bubbles in the shell of the liquid in which the foam generator operates.

It is preferable to apply a mist treatment with a high cavitation effect for thin and precise washing technologies, which occurs when the air bubbles are broken. 
Effective formation of mist requires an air pressure of 7 to 8 bar at a flow rate determined on the basis of the volume of the treated liquid.

14. Aqueous solutions treatment with high levels of pollutants

Made for pretreatment of aqueous solutions before electrochemical or any other treatment.

For processing, the aqueous solution foams and contaminants are concentrated in the foam.

The more pollutants are prone to foam, the more effective the treatment process. Foam removes contaminants.

For effective foam formation requires an air pressure between 6 and 8 bar (the flow rate is determined on the basis of the volume of the treated liquid)

15. Processing of aqueous solutions containing chemical complexes Made for pretreatment of aqueous solutions before electrochemical or any other treatment.

In this case, the foaming of an aqueous solution is used when all impurities are oxidized, and all impurities are in the composition of the foam, since water does not foam.

If the foam is permanently removed, the system comes to a point when all substances that have foam formation characteristics are removed from the solution.

Effective foam formation requires an air pressure of between 6 and 8 bar (the flow rate is determined on the basis of the volume of the treated liquid). 


\title{
References and patent-licensed materials:
}

United States Patent Application

20180186656

Kind Code

Drewniak; Marta ; et al.

July 5, 2018

System and Method for Automated Control, Feed, Delivery Verification, and Inventory Management of Corrosion and Scale Treatment Products for Water Systems

\begin{abstract}
A system and method of controlling the treatment of water systems comprises multiple feeders for separately feeding treatment products, in the form of concentrated, nonhazardous, liquids with a single active ingredient, to a water system to treat various issues, such as corrosion and biofilms. A sensor verifies delivery of the treatment product to the water system. A controller controls activation of each feeder to control a feed rate according to programmed functions. The controller receives signals from sensors, which can be used as inputs in calculating feed rates or feeder activation times according to the programed functions and can alter treatment product feed rates based on real time data regarding water system chemistry or flow rates. The controller can send and receive data, signals, alerts, alarms or changes in programming to or from remote users, remote computers, or a water system controller.
\end{abstract}

Приложение 2

United States Patent Application

20180134554

Kind Code

ALVAREZ, JR.; Daniel ; et al.

May 17, 2018

METHODS AND SYSTEMS FOR PURIFYING HYDROGEN PEROXIDE SOLUTIONS

\begin{abstract}
Compositions, methods, devices, and systems for purifying a source liquid from a replenishment stock solution that includes stabilizing agents, such as metal ions, prior to vaporization. Certain embodiments effect the purification with a solid per-fluoronated ionomer, such as a per-fluoronated ionomer membrane. Advantageously, source liquids purified in this manner provide feed stocks for production of ultra-pure gaseous reagents. As well, performance characteristics of membrane-based vaporizers relying on transport processes are improved.
\end{abstract}


Приложение 3

United States Patent Application

20180128656

Kind Code

Hay; Stephane ; et al.

May 10, 2018

SENSOR

\begin{abstract}
A sensor for operating in an environment subjected to corrosive gases or liquids under pressure, including a housing inside which are installed: a sensing portion, at least one wire electrically connected to the sensing portion, and a seal for sealing the connection between the sensing portion and the at least one wire, with at least one through-hole receiving a portion of the at least one wire. The sensor further including a compressor installed inside the housing, movable with respect to the housing and adapted to contact the seal in order to compress. The seal is made from a single material that is softer than the material constituting the housing. Upon displacement of the compressor, the seal is deformed until the creation of a compression force exerted by the seal onto the portion of the at least one wire, prevents any passage of corrosive gases or liquids between the seal there-between.
\end{abstract}

Приложение 4

United States Patent Application

20180119502

Kind Code

Shanks; David L. ; et al.

May 3, 2018

Method of Capturing Vapor Phase Fluids From Fluid Returns In A Tank

\begin{abstract}
A gas trap system for releasing gas-phase fluids is provided herein. The gas trap system is designed to reside within a return fluids tank, such as at a drill site. The gas trap system first includes a gas trap. The gas trap is configured to agitate drilling fluids in the return tank, and then to release gases during agitation. Liquids are circulated and released through a liquids exhaust port while gases are released through a gas exhaust port near the top of the gas trap. The gas trap system is configured to float along vertical guide rods in response to changes in height, weight and viscosity of the drilling fluids in the return tank. A method of
\end{abstract}


capturing gaseous phase fluids from a fluid return is also provided herein. The fluid return is preferably drilling fluids at a drill site.

Приложение 5

United States Patent Application

20180070866

Kind Code

A1

Raahemifar; Kaamran ; et al.

March 15, 2018

$\boldsymbol{N O} \boldsymbol{N}$-INVASIVE NANOSENSOR SYSTEM TO DETERMINE ANALYTE CONCENTRATION IN BLOOD AND/OR BODILY FLUIDS.

\begin{abstract}
The device is an ultra-low power, $\boldsymbol{n o n}$-invasive in-vivo blood analyte sensor system incorporating multiple sensors including a carbon base and/or carbon base material coated with metallic nanoparticles and/or metallic nanoparticle nanoprobes, as a modified Clark electrode sensor system, that detects hydrogen peroxide concentrations, $\mathrm{pH}$, and/or glucose concentrations (and other analytes) in bodily secretions (e.g. tears, saliva, sweat). The device consists of multiple chemoreceptive sensors, a microprocessor, a signal amplifier, signal filtering, error correction algorithms, analog-to-digital converter and wireless electromagnetic data transmitter to a remote device for further processing and/or data storage (e.g. on a server, on a cloud-based storage system, etc) and/or visual representation via software. The method involves applying the nanoprobe sensor array to skin tissue and the resulting electrical impulses correlate with glucose concentration within liquids such as tears, saliva, blood, etc. The collected data is then represented visually on a computer (handheld, smart-phone, desktop, laptop, etc) via software. The device is powered by ambient electromagnetic radiation, thermoelectric and/or solar power and/or rechargeable battery. The device is placed against the skin or immersed in a sample for sensor measurement. Single and continuous data collection is possible. The device can be reused repeatedly, re-sterilized and it is a high accuracy, low-cost option for multiple use glucose concentration measurements. The device can monitor blood glucose for Type I and Type II diabetics and it is suitable for a wide range of applications including gases, liquids and solids, biological, organic and inorganic chemical analysis.
\end{abstract}

Приложение 6

United States Patent Application

20170328928

Kind Code

Brennen; Reid A. ; et al.

November 16, 2017 


\begin{abstract}
One or more liquids are transferred from a source array to one or more remotely positioned destination sites such as chambers by utilizing one or more movable transfer elements, such as contact pins or capillaries. The source array may include a predetermined organization of addresses at which materials are positioned. One or more materials may be selected for transfer. Based on the selection, one or more addresses may be accessed by the transfer element(s). The addresses may correspond to spots on a surface of the source array. Each spot may be a feature containing one or more (bio)chemical compounds. At the chamber(s), the material(s) may be processed, such by reaction with one or more reagents. The reaction(s) may entail synthesis of one or more desired products. Alternatively, reaction(s) may be performed at the source array, and the product(s) then transferred to the chamber(s).
\end{abstract}

Приложение 7

United States Patent Application

20170269108

Kind Code

Letourneau; Nicolas ; et al.

September 21, 2017

METHODS AND APPARATUS FOR DETECTING COMPOUNDS IN LIQUIDS

\begin{abstract}
Described herein are apparatus and methods for detecting substances of abuse or other analytes in liquids. For example, the apparatus and methods described herein can be used for real-time detection of analytes, such as substances of abuse. The methods comprise providing a detection area comprising a chromatographic membrane capable of receiving the liquid and allowing for migration of the liquid, the chromatographic membrane comprising an antianalyte antibody-particle conjugate, an analyte-conjugate protein at a test line; exposing at least the first location of the apparatus to the liquid; and determining whether an interaction between the analyte-conjugate protein and the liquid occurs to detect the presence of the analyte. The chromatographic membrane may further comprise an anti-species antibody at a control line. Specific buffers are disclosed, and these buffers may be used in the preparation of the apparatus to overcome challenges associated with miniaturization and challenges associated with exposure to beverages.
\end{abstract}

